\title{
Chemoradiotherapy is associated with improved survival for resected pancreatic adenosquamous carcinoma: a retrospective cohort study from the SEER database
}

\author{
Yuan Fang", Ning Pü, Lei Zhang", Wenchuan Wu, Wenhui Lou \\ Department of Pancreatic Surgery, Zhongshan Hospital, Fudan University, Shanghai 200032, China \\ Contributions: (I) Conception and design: W Lou; (II) Administrative support: W Lou; (III) Provision of study materials or patients: N Pu, L Zhang; \\ (IV) Collection and assembly of data: Y Fang, N Pu; (V) Data analysis and interpretation: Y Fang, N Pu; (VI) Manuscript writing: All authors; (VII) \\ Final approval of manuscript: All authors. \\ "These authors contributed equally to this work. \\ Correspondence to: Wenhui Lou, MD; Wenchuan Wu, MD. Department of Pancreatic Surgery, Zhongshan Hospital, Fudan University, 180 Fenglin \\ Road, Shanghai 200032, China. Email: louzsh@126.com; wu.wenchuan@zs-hospital.sh.cn.
}

\begin{abstract}
Background: The prognosis of pancreatic adenosquamous carcinoma (PASC) after surgery is poor. The purpose of this study was to clarify the prognostic factors of PASC and evaluate the efficacy of combination chemoradiotherapy.

Methods: The patients' data retrieved from the Surveillance, Epidemiology, and End Results database (SEER) between 2004 and 2015 were stratified and analyzed in this study. The univariate and multivariate analysis were used for overall survival (OS) and cancer-specific survival (CSS).

Results: T staging, $M$ staging, chemotherapy and radiotherapy is the independent prognostic indicator after PASC resection for both OS and CSS. In the total cohort, 44 patients had both chemo and radiotherapy, with median OS 23 months and CSS 29 months, which was significantly better than neither chemo nor radiotherapy group (68 patients, median OS 8 months and CSS 11 months), and either chemotherapy or radiotherapy group (91 patients, median OS 13 months and CSS 15 months). The survival benefit of chemoradiotherapy was validated in the specific group $(n=159)$ who had only primary PASC. PASC patients receiving chemoradiotherapy had longer OS and CSS than those with neither chemo nor radiotherapy in TNM stage I, II and IV subgroups.

Conclusions: The chemoradiotherapy revealed its prognostic superiority in PASC treatment.
\end{abstract}

Keywords: Pancreatic adenosquamous carcinoma (PASC); adjuvant treatment; chemoradiotherapy

Submitted Jul 17, 2019. Accepted for publication Sep 26, 2019.

doi: $10.21037 /$ atm.2019.10.12

View this article at: http://dx.doi.org/10.21037/atm.2019.10.12

\section{Introduction}

Pancreatic adenosquamous carcinoma (PASC) is a relatively rare histological subtype of pancreatic exocrine neoplasms which is characterized by combinations of two malignant components, including pancreatic ductal adenocarcinoma (PDAC) and squamous cell carcinoma (SCC) (1). It is usually termed by having the SCC component of more than $30 \%$ of the tumor (2).

PASC originates from primary tissues that normally have glandular epithelium, such as stomach, colon, usually represented by a white-gray firm and multinodular mass macroscopically (3), with Cytokeratin 5 (CK-5) and P63 positivity in the squamous components (4).

Previously a large population-based cohort of 415 patients with PASC, including 90 patients who underwent surgical resection, was analyzed from the Surveillance, Epidemiology, and End Results (SEER) database. There is a statistically significant difference between the survival of 
patients with PASC and those with PDAC after resection, with median OS of 12 and 16 months respectively (5).

Traditionally for the PDAC cases with regional invasion, lymph node or vessel metastasis, postoperative comprehensive treatment should be considered $(6,7)$. Despite the accumulation of reports of PASC, most of the treatment experiences have been from case report and small-sample surgical studies (8). Moreover, in the recent years clinical trials were mainly focused on the adjuvant treatment of PDAC, with very limited clinical experiences on the adjuvant treatment in PASC (9), and whether and when there is necessity of adjuvant treatment after resection of PASC is still under debate (10). Due to the dismal prognosis of PASC, when and how to implement the comprehensive treatment is even much more to solve this clinical dilemma. The purpose of this study was therefore to clarify the prognostic factors of PASC patients after surgical resection so as to evaluate the new indication of adjuvant treatment of PASC, which may bring survival benefit to the PASC patients.

\section{Methods}

\section{Patient population and data source}

The data used in this study were retrieved from SEER database registry of National Cancer Institute (https://seer. cancer.gov/). All the data accessed from the SEER database were freely available. The November 2017 submission was used, which had complete information on incidence from 1973 to 2015 and OS or CSS through 2017.

The following criteria were included as another SEER analysis we implemented before $(11,12)$ : all patients were diagnosed as PASC (ICD-O-3: 8560/3) from 2004 to 2015 with surgical resection, along with explicit data about gender, age, primary site, grade, $\mathrm{T}$ staging, $\mathrm{N}$ staging, $M$ staging, postoperative chemotherapy, radiotherapy, number of concomitant tumors and survival information. In addition, patients with unknown demographic, operative or histopathological details, or lack of survival information were excluded in our study.

The AJCC 8th TNM stage was evaluated based on the following codes: collaborative stage (CS) tumor size 2004, CS extension 2004, CS lymph nodes 2004, CS metastases at DX 2004, and derived AJCC stage group (7th edition; 2010+). Overall survival (OS) was calculated by the time from surgery to the last follow-up or death, while cancer specific survival (CSS) was defined as the time from surgery to cancer-related death. The study has been registered in Chinese Clinical Trial Registry. Clinical trial registration number: ChiCTR1900024523.

\section{Statistical analysis}

All statistical analyses were performed by SPSS 21.0 statistical package (IBM Corporation, Armonk, NY, USA). The OS and CSS were compared by Kaplan-Meier curves and analyzed using the log-rank test via GraphPad Prism 6 Software (GraphPad Software Inc., San Diego, CA, USA). The univariate and multivariate analyses and hazard ratios (HRs) were used by Cox proportional hazards regression model to find its independent prognostic risks, and $\mathrm{P}<0.05$ was considered as statistically significant difference.

\section{Results}

\section{Clinicopathological characteristics}

The total of 483 patients diagnosed as PASC from 2004 to 2015 were identified in SEER database. From the criteria mentioned above, 273 patients with no surgical resection were excluded and 3 patients were excluded because of unknown surgery information. In addition, 2 patients with unknown tumor size, 1 patient with unknown lymph node metastasis and 1 patient with unknown distant metastasis were all excluded. So finally, 203 patients with histologically confirmed PASC from the SEER database were finally included, with the detailed baseline characteristics showed in Table 1. There are 108 female and 95 male patients with the median age of 70 years (range, 39-89 years). Tumor located 108 at pancreatic head and 95 at body or tail. About $26(13 \%), 48(24 \%), 122(60 \%)$ and $7(3 \%)$ patients were undefined, moderate (Grade II), poor (Grade III) and undifferentiated differentiations (Grade IV) respectively. Among the 203 patients, T1 to T4 classification were 7, 85,73 and 38 cases respectively; 126 (62\%) of PASC were lymph node positive, 18 patients had distant metastasis that may be enrolled in clinical trials, 8 of which were liver metastasis. According to AJCC 8th staging system, number of stage I, II, III, IV was 36, 89, 60, 18 respectively. A total of 159 patients had only one primary cancer (PASC), while 44 patients had other metachronous primary tumors.

\section{Prognostic factors of OS and CSS}

In the total cohort, the median OS was 12 months (range, 
Table 1 The clinicopathological stigma and multivariate analysis in total cohort

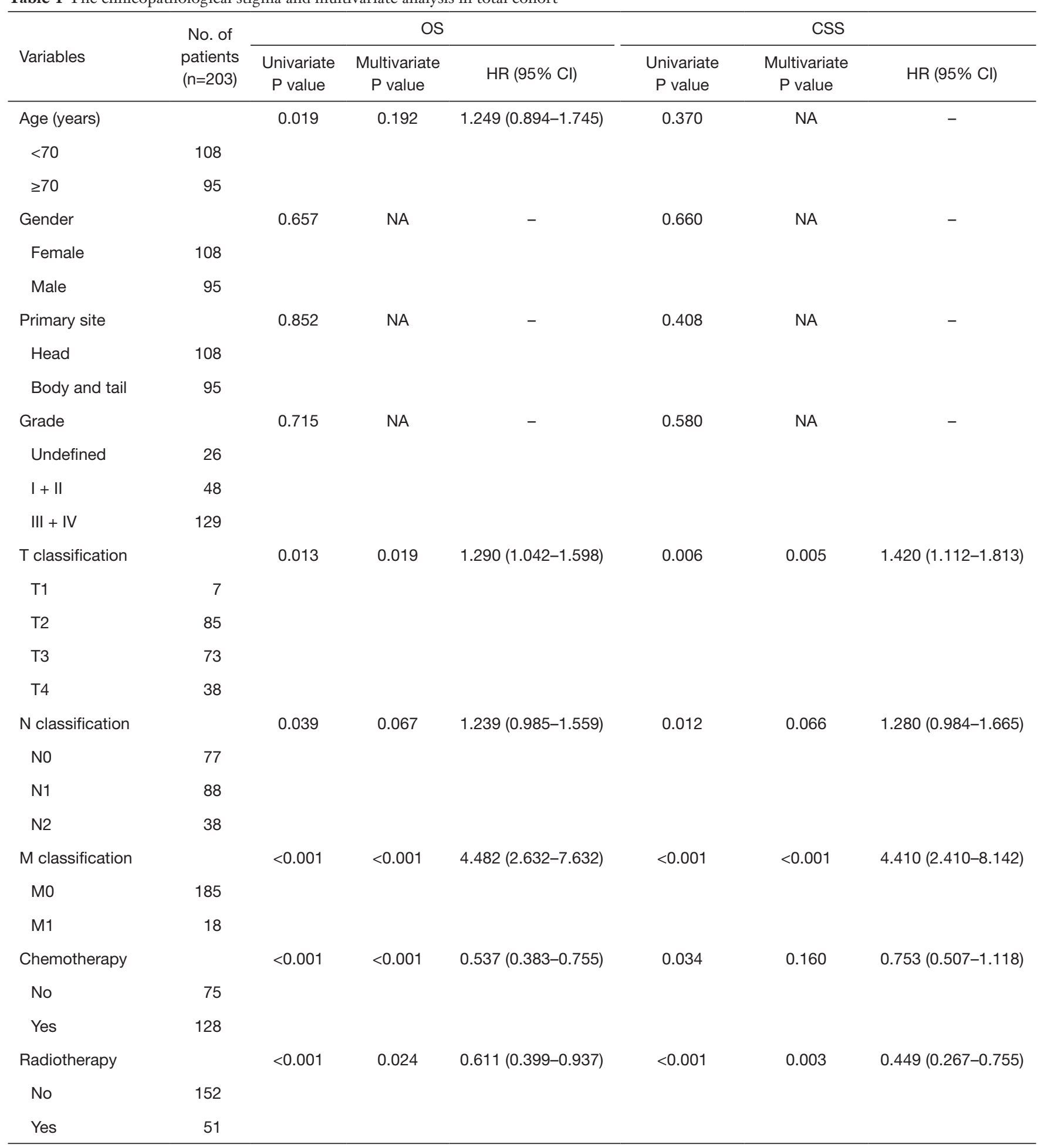

OS, overall survival; CSS, cancer-specific survival; NA, not applicable. 

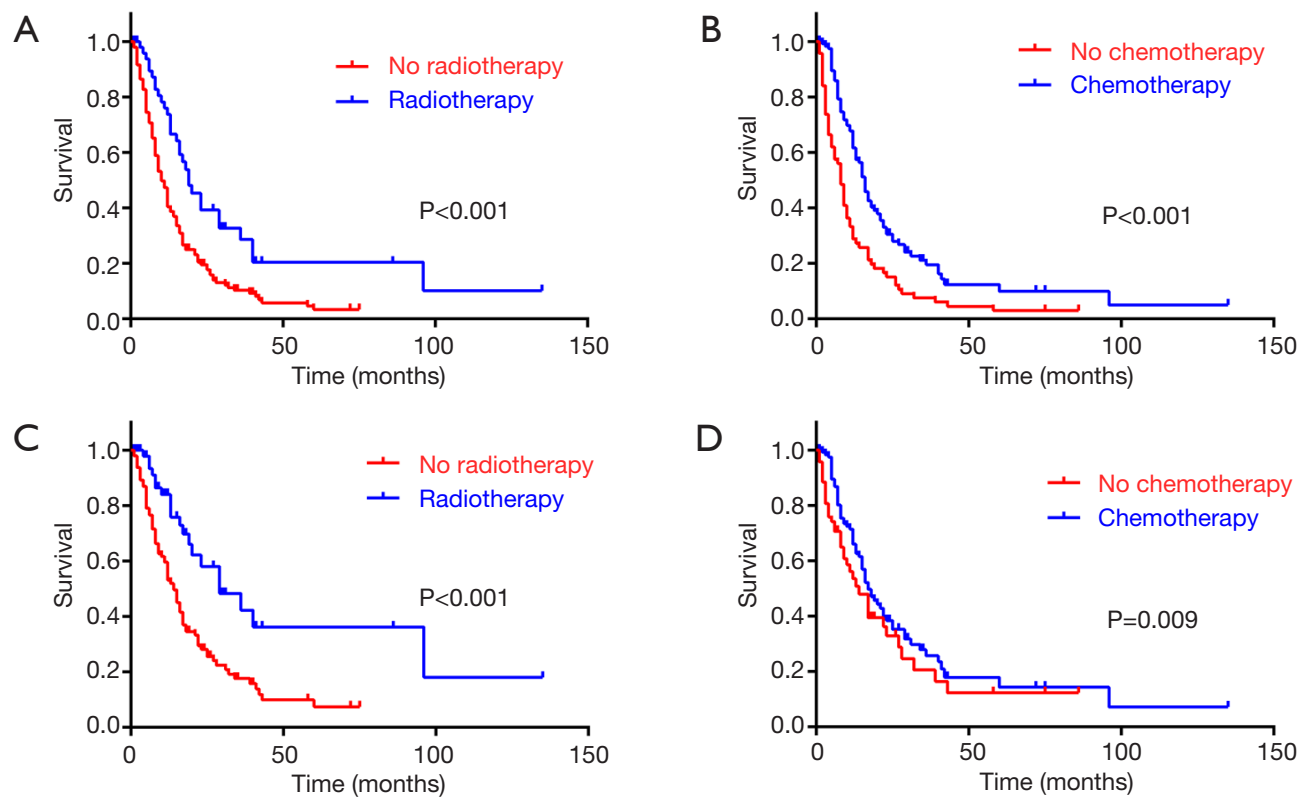

Figure 1 Radiotherapy (A) and chemotherapy (B) were prognostic indicators in OS, and radiotherapy (C) and chemotherapy (D) were prognostic indicators in CSS. OS, overall survival; CSS, cancer-specific survival.

1-135 months), 1-, 3- and 5-year OS rates were $48.1 \%$, $14.5 \%$ and $6.8 \%$ respectively. Considering the influence of other metachronous primary tumors on survival, CSS was applied to calculate the survival from surgery for PASC to death caused from PASC. So, the median CSS was 16 months, 1-, 3- and 5-year CSS rates were 60.5\%, 23.6\%, $13.5 \%$ respectively. As it is showed in univariate analysis, older age $\geq 70(\mathrm{P}=0.019)$, $\mathrm{T}$ staging $(\mathrm{P}=0.013), \mathrm{N}$ staging $(\mathrm{P}=0.039), M$ staging $(\mathrm{P}<0.001)$, radiotherapy $(\mathrm{P}<0.001)$ and chemotherapy $(\mathrm{P}<0.001)$ were significant risk factors of OS (Table 1). In multivariate analysis for OS, T staging $(\mathrm{P}=0.019$, HR 1.290, 95\% CI: 1.042-1.598), M staging $(\mathrm{P}<0.001$, HR 4.482, 95\% CI: 2.632-7.632) (Table 1), radiotherapy $(\mathrm{P}=0.024$, HR 0.611, 95\% CI: 0.399-0.937) and chemotherapy $(\mathrm{P}<0.001$, HR $0.537,95 \%$ CI: 0.383-0.755) were independent prognostic indicators (Figure $1 A, B)$.

As to the CSS, advanced $\mathrm{T}$ staging $(\mathrm{P}=0.006), \mathrm{N}$ staging $(\mathrm{P}=0.012), M$ staging $(\mathrm{P}<0.001)$, radiotherapy $(\mathrm{P}<0.001)$ and chemotherapy $(\mathrm{P}=0.034)$ were significant risk factors (Table 1), with advanced T staging $(\mathrm{P}=0.005$; HR 1.420; 95\% CI: $1.112-1.813), M$ staging $(\mathrm{P}<0.001$, HR 4.410, 95\% CI: $2.410-8.142)$, and radiotherapy $(\mathrm{P}=0.003, \mathrm{HR}$ 0.449, 95\% CI: $0.267-0.755)$ were independent prognostic indicators (Figure $1 C, D$ ). Although the insignificant $\mathrm{p}$ value of chemotherapy in the multivariate analysis for CSS $(\mathrm{P}=0.160)$, it still had a potential role in PASC treatment according to $0.753 \mathrm{HR}$ value. In view of the results above, TNM 8th staging system (T staging and $M$ staging), chemotherapy and radiotherapy may be the indicator for survival benefit after PASC resection.

\section{Role of chemotherapy and radiotherapy in the prognosis of resectable PASC}

Among the 203 patients, 68 patients had neither chemo nor radiotherapy, with their median OS 8 months, median CSS 11 months. 91 patients had single therapy (either post-operative chemotherapy or radiotherapy), with their median OS 13 months, median CSS 15 months; 44 patients had both post-operative chemo and radiotherapy, with their median OS 23 months, median CSS 29 months. As the Kaplan-Meier curves showed, the patients of chemoradiotherapy group had much better prognosis, which was further confirmed by the absolutely HRs with statistical significance (Table 2) and the combined chemo and radiotherapy had the excellent survival outcome compared with another two groups (Figure 2). In addition, there was no correlation between adjuvant treatment regimen and other clinicopathological characteristics (Table 3).

Then, whether the prognostic benefit of single chemotherapy is superior to single radiotherapy was elucidated. Among the single therapy subgroup, 72 patients 
Table 2 The survival benefit of chemoradiotherapy compared to other treatment by univariate analysis

\begin{tabular}{|c|c|c|c|c|c|}
\hline Variables & $\begin{array}{l}\text { No. of patients } \\
\quad(n=203)\end{array}$ & \multicolumn{2}{|r|}{ OS } & \multicolumn{2}{|c|}{ CSS } \\
\hline Chemoradiotherapy & 44 & Reference & & Reference & \\
\hline Single therapy & 91 & 0.012 & $1.822(1.138-2.916)$ & 0.006 & $2.163(1.252-3.736)$ \\
\hline No chemoradiotherapy & 68 & $<0.001$ & 3.247 (2.026-5.202) & $<0.001$ & $2.924(1.656-5.161)$ \\
\hline
\end{tabular}

OS, overall survival; CSS, cancer-specific survival.
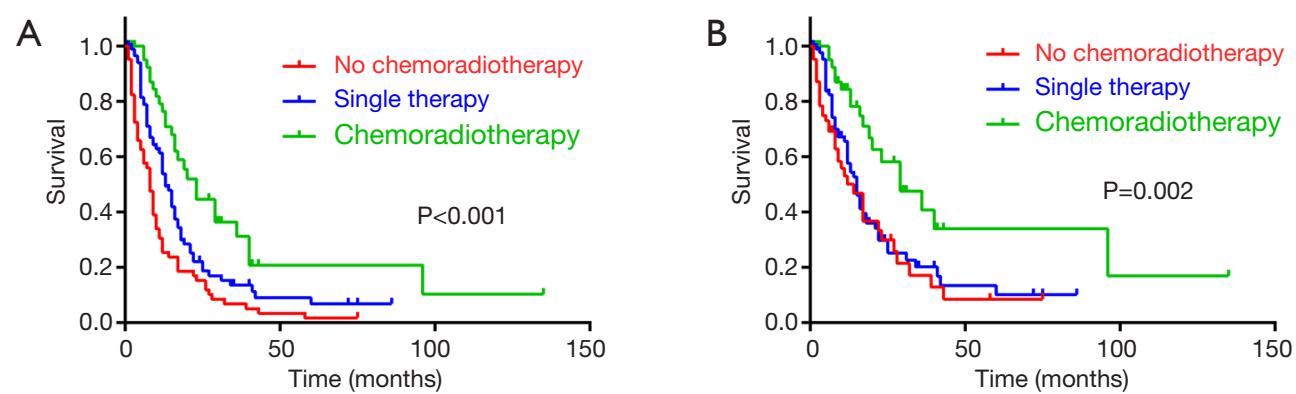

Figure 2 Combined chemoradiotherapy had the better survival compared with single therapy group and no chemoradiotherapy groups in OS (A) and CSS (B). OS, overall survival; CSS, cancer-specific survival.

had single chemotherapy, while only 5 patients had single radiotherapy. In the single chemotherapy subgroup, the median OS was 12 months, while in single radiotherapy subgroup the median OS was 13 months (Log-Rank test $\mathrm{P}=0.582$ ). Concomitantly, the median CSS of single chemotherapy subgroup was 12 months, while it was not available in single radiotherapy subgroup (Log-Rank test $\mathrm{P}=0.164)$. Therefore, there is no difference of survival between chemotherapy and radiotherapy subgroups, but the survival of combined chemoradiotherapy was superior to single therapy.

\section{The validation of chemoradiotherapy in the specific group} of PASC

Owing to the concurrence of other tumors in the total 203 patients, the efficacy of chemoradiotherapy may be biased. So, we analyzed the 159 patients who had only the primary cancer of PASC. There were 47 patients had neither chemo nor radiotherapy, with the median OS 5 months, median CSS 6 months, 77 patients had single therapy, with median OS 12 months, median CSS 13 months and 35 patients had combined chemoradiotherapy, with the median OS 23 months, median CSS 29 months (both Log Rank test $\mathrm{P}<0.001$ ) (Figure 3). Through the multivariate analysis of OS and CSS, M staging, chemotherapy and radiotherapy were confirmed as the independent risk factors for resectable PASC (Table 4). In the whole specific cohort, patients with no chemoradiotherapy or single therapy showed a significant shorter survival and higher risk than those with chemoradiotherapy in OS $(\mathrm{P}<0.001$, HR 3.385, 95\% CI: $1.953-5.867$; $\mathrm{P}=0.007$, HR 2.088, 95\% CI: $1.225-$ 3.560) and CSS ( $\mathrm{P}<0.001$, HR 3.466, 95\% CI: 1.938-6.198; $\mathrm{P}=0.006$, HR $2.189,95 \%$ CI: $1.247-3.842)$.

\section{AfCC 8th staging system based chemoradiotherapy survival analysis}

Based on the specific PASC cohort, 159 patients were divided into four subgroups according to AJCC 8th staging system. There were 27, 69, 49 and 14 patients in stage I, II, III and IV respectively. There were significant differences from stage I to IV in terms of OS and CSS (Table 5, Figure 4). However, the HRs in stage II, III and IV were 1.360, 1.664 and 5.697 of OS, and 1.343, 1.761 and 6.565 of CSS in reference to stage I. This also revealed that PASC patients with higher TNM stage might had higher risk and poorer survival. In TNM stage subgroups, the univariate analysis showed that PASC patients receiving chemoradiotherapy had longer OS and CSS than those with neither chemo nor 
Table 3 Correlation between adjuvant treatment and clinicopathological characteristics of patients with PASC

\begin{tabular}{lccc}
\hline \multirow{2}{*}{ Variables } & \multicolumn{3}{c}{ Adjuvant treatment } \\
\cline { 2 - 4 } & No chemoradiotherapy & Single therapy & Chemoradiotherapy \\
\hline Primary site (head/body and tail) & $34 / 34$ & $52 / 39$ & $22 / 22$ \\
Grade (unknown/I + II/III + IV) & $9 / 19 / 40$ & $8 / 20 / 63$ & $9 / 9 / 26$ \\
T classification (T1/T2/T3/T4) & $2 / 24 / 30 / 12$ & $4 / 42 / 30 / 15$ & $1 / 19 / 13 / 11$ \\
N classification (N0/N1/N2) & $29 / 26 / 13$ & $28 / 41 / 22$ & $20 / 21 / 3$ \\
M classification (M0/M1) & $63 / 5$ & $80 / 11$ & $42 / 2$ \\
\hline
\end{tabular}

OS, overall survival; CSS, cancer-specific survival; PASC, pancreatic adenosquamous carcinoma.
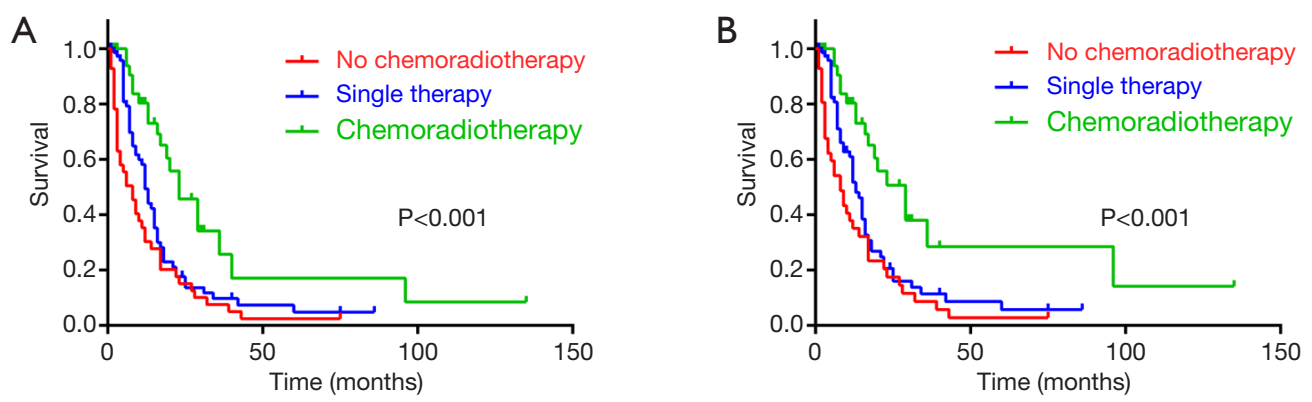

Figure 3 Combined chemoradiotherapy had the better survival compared with single therapy group and no chemoradiotherapy groups in OS (A) and CSS (B) in the specific group with only primary PASC. OS, overall survival; CSS, cancer-specific survival; PASC, pancreatic adenosquamous carcinoma.

radiotherapy in stage I, II and IV subgroups. Owing to the limited cases in each subgroup, it showed no significant difference in chemoradiotherapy subgroup and single therapy subgroup, but the HRs in single therapy subgroup of OS and CSS were about 1-2.5 times higher than those in chemoradiotherapy subgroup. All of these results revealed that the chemoradiotherapy may had its potential superiority in PASC treatment.

\section{Discussion}

Pancreatic cancer is the fourth leading cause of cancerrelated deaths in the United States with 56,770 new cases and 45,750 deaths estimated in $2019(13,14)$. PASC was classified as one subtype of PDAC, according to the 2010 WHO classification (15). In our study, a total of 203 patients with resected PASC from SEER database were enrolled and analyzed. PASC comprising $4 \%$ of exocrine pancreatic malignancies (16), has been considered to show even much worse prognosis than PDAC (17). But previous studies about PASC have shown several problems. One is the staging of the enrollment of the disease in that the reports have mainly mentioned early-stage disease, instead of metastatic disease (18). The other is that due to the rarity of PASC cases compared to PDAC, the clinical features of PASC were mainly based on case report or small-sample studies $(19,20)$. Besides, there was also one SEER analysis (5) comparing the survival following surgical resection in patients with adenosquamous carcinoma $(n=415)$ or adenocarcinoma $(n=45,693)$ and focused on comparing the biological behaviour, survival of PASC with PDAC, together with the surgical benefit of the survival, while the prognostic benefit of surgery was not satisfactory enough. Therefore, very little is known about which prognostic factors are associated with PASC and which kind of adjuvant treatment is needed for promoting the poor prognosis of PASC (21).

From the results of our study, the 203 resected PASC patients have a poor prognosis (total median OS, 12 months, 1-, 3- and 5-year OS were $48.1 \%, 14.5 \%$ and $6.8 \%$ respectively, median CSS, 16 months, 1-, 3- and 5 -year CSS were $60.5 \%, 23.6 \%, 13.5 \%$ respectively). 
Table 4 The clinicopathological stigma and multivariate analysis in specific PAC cohort

\begin{tabular}{|c|c|c|c|c|c|c|c|}
\hline Variables & $\begin{array}{l}\text { No. of patients } \\
\qquad(n=203)\end{array}$ & \multicolumn{3}{|c|}{ OS } & \multicolumn{3}{|c|}{ CSS } \\
\hline Age (years) & & 0.090 & NA & - & 0.272 & NA & - \\
\hline$<70$ & 96 & & & & & & \\
\hline Female & 83 & & & & & & \\
\hline Male & 76 & & & & & & \\
\hline Primary site & & 0.817 & NA & - & 0.602 & NA & - \\
\hline Unknown & 20 & & & & & & \\
\hline$I+I I$ & 40 & & & & & & \\
\hline III + IV & 99 & & & & & & \\
\hline T classification & & 0.048 & 0.072 & $1.254(0.980-1.605)$ & 0.054 & NA & - \\
\hline $\mathrm{T} 1$ & 5 & & & & & & \\
\hline $\mathrm{T} 2$ & 64 & & & & & & \\
\hline T3 & 60 & & & & & & \\
\hline M classification & & $<0.001$ & $<0.001$ & $4.391(2.376-8.114)$ & $<0.001$ & $<0.001$ & $5.232(2.814-9.727)$ \\
\hline MO & 145 & & & & & & \\
\hline M1 & 14 & & & & & & \\
\hline Chemotherapy & & $<0.001$ & 0.006 & $0.581(0.395-0.855)$ & 0.005 & 0.022 & $0.623(0.416-0.935)$ \\
\hline No & 52 & & & & & & \\
\hline Yes & 107 & & & & & & \\
\hline Radiotherapy & & $<0.001$ & 0.002 & $0.473(0.293-0.764)$ & $<0.001$ & 0.001 & $0.407(0.242-0.683)$ \\
\hline No & 119 & & & & & & \\
\hline Yes & 40 & & & & & & \\
\hline
\end{tabular}

OS, overall survival; CSS, cancer-specific survival; NA, not applicable. 
Table 5 The univariate analysis of chemoradiotherapy based on AJCC 8th staging system

\begin{tabular}{|c|c|c|c|c|c|}
\hline Variables & $\begin{array}{l}\text { No. of patients } \\
\qquad(n=159)\end{array}$ & \multicolumn{2}{|r|}{ os } & \multicolumn{2}{|r|}{ css } \\
\hline TNM stage I & 27 & Reference & & Reference & \\
\hline Chemoradiotherapy & 9 & 1 & & 1 & \\
\hline Single therapy & 9 & 0.919 & $1.068(0.299-3.823)$ & 0.636 & $1.376(0.367-5.158)$ \\
\hline TNM stage II & 69 & 0.253 & $1.360(0.802-2.305)$ & 0.301 & $1.343(0.768-2.347)$ \\
\hline Chemoradiotherapy & 15 & 1 & & 1 & \\
\hline Single therapy & 32 & 0.144 & $2.060(0.840-5.050)$ & 0.153 & $2.046(0.766-5.464)$ \\
\hline No chemoradiotherapy & 22 & $0.003^{*}$ & 3.927 (1.577-9.779) & $0.003^{*}$ & $4.466(1.666-11.967)$ \\
\hline Single therapy & 27 & 0.126 & $2.330(0.789-6.878)$ & 0.128 & $2.317(0.784-6.846)$ \\
\hline No chemoradiotherapy & 13 & 0.068 & 2.949 (0.922-9.429) & 0.162 & $2.355(0.710-7.815)$ \\
\hline TNM stage IV & 14 & $<0.001^{\#}$ & $5.697(2.729-11.892)$ & $<0.001^{\#}$ & $6.565(3.080-13.996)$ \\
\hline Chemoradiotherapy & 2 & 1 & & 1 & \\
\hline Single therapy & 9 & 0.381 & $2.070(0.407-10.523)$ & 0.381 & $2.070(0.407-10.523)$ \\
\hline No chemoradiotherapy & 3 & $0.017^{\star}$ & $26.672(1.798-395.590)$ & $0.017^{*}$ & $26.672(1.798-395.590)$ \\
\hline
\end{tabular}

*, significant differences in treatment subgroups of each stage; ", significant differences in AJCC TNM staging system. OS, overall survival; CSS, cancer-specific survival.
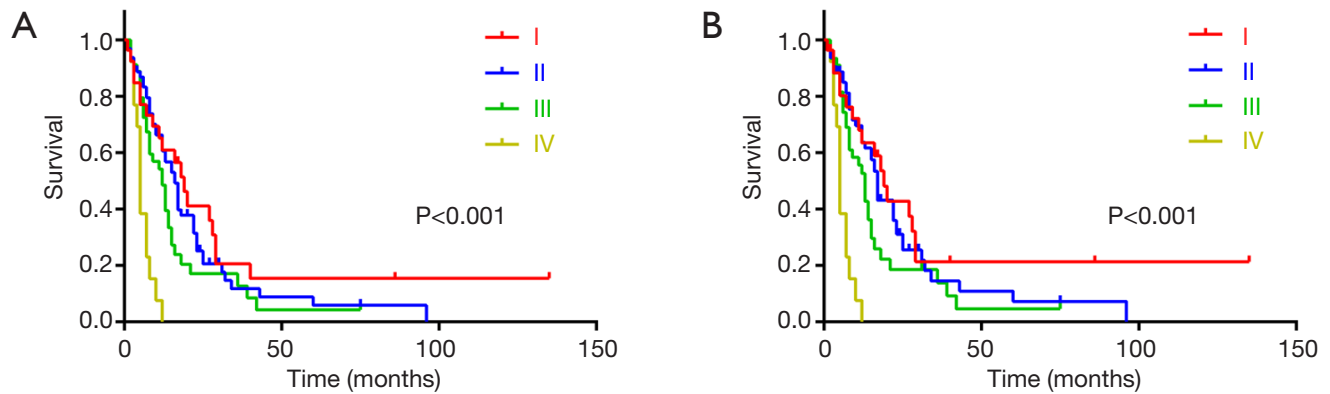

Figure 4 There were significant differences from stage I to IV in terms of OS (A) and CSS (B). OS, overall survival; CSS, cancer-specific survival.

This survival is approximate to the result from the largest single institution of Johns Hopkins Hospital (median OS: 10.9 months) (22).

In light of the very poor prognosis of PASC, we discovered that $\mathrm{T}$ staging, $\mathrm{M}$ staging and adjuvant treatment including chemo and radiotherapy may be the indicator for survival benefit after PASC resection. Besides, it is a remarkable fact that patients who received adjuvant treatment had a much better survival compared with those without treatment. Among the 203 patients, 91 had either chemo or radiotherapy, with their median OS 13 months, median CSS 15 months; in patients with both chemo and radiotherapy, the survival data is even much better than either of the single treatment, with the median OS and CSS at 23 and 29 months respectively. As the Kaplan-Meier curves showed, the patients of 
chemoradiotherapy group had much better prognosis, which was further confirmed by the absolutely HRs with statistical significance. Moreover, in the specific cohort (159 CSS group), patients with no chemoradiotherapy or single therapy showed a significant shorter survival and higher risk than those with chemoradiotherapy in OS and CSS. All these results remind us that for improving the poor prognosis of PASC, the comprehensive treatment (i.e., Combined chemoradiotherapy), instead of only chemotherapy or radiotherapy, is much more prognosticbenefit than the normal PDAC. However, the treatment paradigm of how to choose chemotherapy or radiotherapy, and how to choose neochemoradiotherapy or postoperative chemoradiotherapy is still need to be further elucidated.

There are some limitations of our study. Given it is from the SEER database, the detail of the surgery and postoperative treatment, such as resection margin, patterns of recurrence, specific medical regimen of the adjuvant therapy was not available. So, we cannot compare the efficacy between different specific chemoradiation regimen and identify the optimal treatment. Besides, it is interesting that platinum-based agents have demonstrated improved outcomes in squamous cell cancer such as the esophagus cancer and head and neck cancer (23), while the efficacy of platinum-based agents combined with gemcitabine for PASC was theoretically more promising but only have been limited to some case reports (24).

Accordingly, considering the small sample size of PASC in each pancreatic center, also for elaborating the significance of surgery and adjuvant treatment for the prognosis improvement of PASC, we need more multiinstitutional, prospective, randomized and standard chemoradiation regimen clinical trial to implement the most-beneficial treatment and identify which subgroup of the patients can benefit the most from adjuvant chemoradiation.

\section{Acknowledgments}

Funding: The study was supported by National Natural Science Foundation of China (No. 81702964).

\section{Footnote}

Conflicts of Interest: The authors have no conflicts of interest to declare.

Ethical Statement: The authors are accountable for all aspects of the work in ensuring that questions related to the accuracy or integrity of any part of the work are appropriately investigated and resolved. The data used in this study were retrieved from SEER database registry of National Cancer Institute.

\section{References}

1. Hester CA, Augustine MM, Choti MA, et al. Comparative outcomes of adenosquamous carcinoma of the pancreas: An analysis of the National Cancer Database. J Surg Oncol 2018;118:21-30.

2. Komatsu H, Egawa S, Motoi F, et al. Clinicopathological features and surgical outcomes of adenosquamous carcinoma of the pancreas: a retrospective analysis of patients with resectable stage tumors. Surg Today 2015;45:297-304.

3. Luchini C, Capelli P, Scarpa A. Pancreatic Ductal Adenocarcinoma and Its Variants. Surg Pathol Clin 2016;9:547-60.

4. Marcus R, Maitra A, Roszik J. Recent advances in genomic profiling of adenosquamous carcinoma of the pancreas. J Pathol 2017;243:271-2.

5. Boyd CA, Benarroch-Gampel J, Sheffield KM, et al. 415 patients with adenosquamous carcinoma of the pancreas: a population-based analysis of prognosis and survival. J Surg Res 2012;174:12-9.

6. Neoptolemos JP, Dunn JA, Stocken DD, et al. Adjuvant chemoradiotherapy and chemotherapy in resectable pancreatic cancer: a randomised controlled trial. Lancet 2001;358:1576-85.

7. Moore MJ, Goldstein D, Hamm J, et al. Erlotinib plus gemcitabine compared with gemcitabine alone in patients with advanced pancreatic cancer: a phase III trial of the National Cancer Institute of Canada Clinical Trials Group. J Clin Oncol 2007;25:1960-6.

8. Regi P, Butturini G, Malleo G, et al. Clinicopathological features of adenosquamous pancreatic cancer. Langenbecks Arch Surg 2011;396:217-22.

9. Hsu JT, Chen HM, Wu RC, et al. Clinicopathologic features and outcomes following surgery for pancreatic adenosquamous carcinoma. World J Surg Oncol 2008;6:95.

10. Shibagaki K, Fujita K, Nakayama S, et al. Complete response of a pancreatic adenosquamous carcinoma to chemoradiotherapy. Int J Clin Oncol 2008;13:74-7.

11. Pu N, Li J, Xu Y, et al. Comparison of prognostic prediction between nomogram based on lymph node ratio and AJCC 8th staging system for patients with resected 
pancreatic head carcinoma: a SEER analysis. Cancer Manag Res 2018;10:227-38.

12. $\mathrm{Pu} \mathrm{N}, \mathrm{Lv} \mathrm{Y}$, Zhao G, et al. Survival prediction in pancreatic cancer patients with no distant metastasis: a large-scale population-based estimate. Future Oncol 2018;14:165-75.

13. Siegel RL, Miller KD, Jemal A. Cancer statistics, 2019. CA Cancer J Clin 2019;69:7-34.

14. Pu N, Gao S, Yin H, et al. Cell-intrinsic PD-1 promotes proliferation in pancreatic cancer by targeting CYR61/CTGF via the hippo pathway. Cancer Lett 2019;460:42-53.

15. Hruban RH, Fukushima N. Pancreatic adenocarcinoma: update on the surgical pathology of carcinomas of ductal origin and PanINs. Mod Pathol 2007;20 Suppl 1:S61-70.

16. Simone CG, Zuluaga Toro T, Chan E, et al. Characteristics and outcomes of adenosquamous carcinoma of the pancreas. Gastrointest Cancer Res 2013;6:75-9.

17. Imaoka H, Shimizu Y, Mizuno N, et al. Clinical characteristics of adenosquamous carcinoma of the pancreas: a matched case-control study. Pancreas 2014;43:287-90.

18. Voong KR, Davison J, Pawlik TM, et al. Resected pancreatic adenosquamous carcinoma: clinicopathologic review and evaluation of adjuvant chemotherapy and radiation in 38 patients. Hum Pathol 2010;41:113-22.

Cite this article as: Fang $\mathrm{Y}, \mathrm{Pu} \mathrm{N}$, Zhang $\mathrm{L}, \mathrm{Wu} \mathrm{W}$, Lou W. Chemoradiotherapy is associated with improved survival for resected pancreatic adenosquamous carcinoma: a retrospective cohort study from the SEER database. Ann Transl Med 2019;7(20):522. doi: 10.21037/atm.2019.10.12
19. Yamaue H, Tanimura H, Onishi $\mathrm{H}$, et al. Adenosquamous carcinoma of the pancreas: successful treatment with extended radical surgery, intraoperative radiation therapy, and locoregional chemotherapy. Int J Pancreatol 2001;29:53-8.

20. Smoot RL, Zhang L, Sebo TJ, et al. Adenosquamous carcinoma of the pancreas: a single-institution experience comparing resection and palliative care. J Am Coll Surg 2008;207:368-70.

21. Okabayashi T, Hanazaki K. Surgical outcome of adenosquamous carcinoma of the pancreas. World J Gastroenterol 2008;14:6765-70.

22. Katz MH, Taylor TH, Al-Refaie WB, et al. Adenosquamous versus adenocarcinoma of the pancreas: a population-based outcomes analysis. J Gastrointest Surg 2011;15:165-74.

23. Yang LY, Li L, Jiang H, et al. Expression of ERCC1 antisense RNA abrogates gemicitabine-mediated cytotoxic synergism with cisplatin in human colon tumor cells defective in mismatch repair but proficient in nucleotide excision repair. Clin Cancer Res 2000;6:773-81.

24. Wild AT, Dholakia AS, Fan KY, et al. Efficacy of platinum chemotherapy agents in the adjuvant setting for adenosquamous carcinoma of the pancreas. J Gastrointest Oncol 2015;6:115-25. 